\title{
REVIEW
}

\section{Mexazolam: Clinical Efficacy and Tolerability in the Treatment of Anxiety}

Hélder Fernandes $\cdot$ Ricardo Moreira

To view enhanced content go to www.neurologytherapy-open.com

Received: March 13, 2014 / Published online: March 29, 2014

(c) The Author(s) 2014. This article is published with open access at Springerlink.com

\section{ABSTRACT}

Introduction: Mexazolam is indicated for the management of anxiety with or without psychoneurotic conditions. In adult patients, the recommended daily dosage of mexazolam is 1-3 mg, administered three times daily. The objective of this article is to review the available information on the benzodiazepine (BZD) mexazolam and its clinical utility in treating patients with anxiety.

Methods: The PubMed database was searched using the keyword "mexazolam" with no date or language restrictions applied to the search. As only 11 papers were retrieved, some previously

Electronic supplementary material The online version of this article (doi:10.1007/s40120-014-0016-7) contains supplementary material, which is available to authorized users.

H. Fernandes $(\bowtie)$

Department of Research and Development, BialPortela \& $C^{\text {a }}$, S.A, S. Mamede do Coronado, Portugal e-mail: helder.fernandes@bial.com

R. Moreira

Clinic of Psychiatry and Mental Health, São João

Hospital Centre, Porto, Portugal

R. Moreira

Department of Clinical Neurosciences and Mental Health, Faculty of Medicine, University of Porto, Porto, Portugal published manuscripts of interest known by the authors (not indexed on PubMed) have been added for completeness. Relevant information was selected for inclusion by the authors.

Results: A number of early studies demonstrated the ability of mexazolam to reduce anxiety symptoms with few side effects in patients with disorders associated with anxiety. Following on from this preliminary evidence, controlled studies directly comparing mexazolam with other BZDs showed that the drug is more effective than bromazepam and oxazolam, and is at least as effective as alprazolam. A larger, multicenter, phase IV study also showed that mexazolam 2 or $3 \mathrm{mg} /$ day rapidly improved Hamilton Anxiety Rating Scale scores and substantially reduced the frequency and severity of numerous somatic anxiety symptoms in patients with anxiety disorders. With regard to safety, the clinical evidence indicates that mexazolam is generally well tolerated, with a low incidence of drowsiness and sedation. Furthermore, the lack of psychomotor or cognitive performance impairment following mexazolam administration may lead to better treatment compliance. 
Conclusion: The available clinical evidence suggests that mexazolam is an effective therapeutic option for the management of anxiety. However, larger, well-controlled clinical trials are needed to directly compare and contrast mexazolam's efficacy and safety with other BZDs.

Keywords: Anxiety; Benzodiazepine; Efficacy; Mexazolam; Psychiatry; Psychomotor and cognitive performance; Tolerability

\section{INTRODUCTION}

Epidemiological research has indicated that anxiety disorders are among the most common psychiatric disorders in the United States [1-3]. Furthermore, data from a World Health Organization World Mental Health survey reported anxiety disorders to be the most common mental disorder (estimated lifetime prevalence of $4.8-31.0 \%$ ) in numerous countries across Africa, Asia, the Americas, Europe, and the Middle East [4].

The most commonly occurring anxiety disorders include generalized anxiety disorder, panic attacks, panic disorder, specific phobia, social anxiety disorder, agoraphobia, traumaand stressor-related disorders, obsessivecompulsive and related disorders, separation anxiety disorder, and illness anxiety disorder [5]. These disorders are marked by excessive fear, anxiety, and associated avoidance behaviors, but are distinguished from each other by the types of objects or situations that induce the fear or avoidance. Anxiety disorders are marked by persistence, rather than transient fear or anxiety, and tend to have their onset in childhood or adolescence (Diagnostic and Statistical Manual of Mental Disorders [DSM]-5) [5].
Following an accurate diagnosis, the recommended treatment options for anxiety disorders include psychotherapies and pharmacological therapies [6-8]. Benzodiazepines (BZDs) are a class of drugs that bind to specific BZD-type receptors on the gamma-aminobutyric acid (GABA) chloride ion complex and facilitate GABA inhibitory effects $[9$, 10]. Although BZDs are not generally recommended for first-line, long-term management of anxiety disorders in clinical practice, their use as second-line treatment is widespread [11].

In 1956, Dr. Leo Sternbach discovered the first BZD, chlordiazepoxide (approved in 1960), which was followed by the release of its congener, diazepam, in 1963 [11]. Since the release of chlordiazepoxide and diazepam, more than 1,000 BZDs have been synthesized. [11]. More recently, four non-BZD hypnotics (zaleplon, zolpidem, zopiclone, and eszopiclone), also known as the so-called z-drugs, were introduced. Although these compounds are effective for the initial treatment of insomnia, their clinical effects are not sustained through the night [11].

Today, there are a wide range of BZDs available for use in clinical practice. Despite sharing the same mechanism of action, they vary in their duration of action (short- vs. longacting) and difference in pharmacokinetic profiles translate, in clinical practice, into differences in their main therapeutic effect (anxiolytic vs. hypnotic) $[11,12]$. BZDs with a strong anxiolytic effect at therapeutic doses, such as diazepam, are effective for the relief of anxiety states, while BZDs with a predominant hypnotic effect, such as flurazepam, are used for the short-term treatment of insomnia [11]. BZDs such as clonazepam are also associated with anticonvulsant effects [13]. 
In addition to their clinical effectiveness, one of the main advantages of BZDs in the treatment of anxiety is their early onset of action [14], which may explain why these agents continue to be widely prescribed. However, BZDs may be associated with a number of adverse effects, particularly at higher doses, which include sedation, physical and psychic dependence, and impaired concentration, memory and psychomotor performance [14]. Furthermore, withdrawal symptoms and rebound anxiety may be associated with the long-term use of these agents [14]. The frequency and severity of these adverse events may also vary between BZDs. BZDs have also been linked to substance abuse/misuse and dependence when used longterm [15].

With the current availability of a variety of BZDs, clinicians are frequently faced with difficulties in choosing the most appropriate BZD for an individual patient. Therefore, it is important for clinicians to have a good understanding of the main characteristics and differences of these drugs. The BZD mexazolam, although currently used in the treatment of anxiety disorders, is not well known and clinical publications are limited and so, in this context, the objective of this article is to review the available information on mexazolam and its clinical utility in treating patients with anxiety.

\section{METHODS}

The PubMed database was searched for articles using the keyword "mexazolam". Only 11 results were retrieved. In order to ensure the review article was as comprehensive as possible, some previously published manuscripts of interest known by the authors have been added. Subsequently, the authors have selected the most relevant information (all preclinical and clinical data, determinant to the interpretation of the several characteristics of mexazolam discussed in the article, were considered to be relevant information by the authors). No date or language restrictions were applied to the search.

The analysis in this article is based on previously conducted studies, and does not involve any new studies of human or animal subjects performed by any of the authors.

\section{MEXAZOLAM}

Mexazolam (also known as CS-386) is currently indicated for the management of anxiety with or without psychoneurotic conditions when the disorder is severe, disabling, or subjecting the individual to extreme distress [16]. The duration of treatment should be as short as possible and the dosage of mexazolam should be adjusted based on an individual patient's age and the severity of symptoms. In adults, the average daily dose is $1-3 \mathrm{mg}$, which is preferably administered three times daily (TID); the daily dosage should not exceed $1.5 \mathrm{mg}$ in elderly patients. Mexazolam is not indicated for use in pediatric patients [16].

\section{Chemical Properties}

Mexazolam (10-chloro-11b-(2-chlorophenyl)-3methyl-2,3,7,11b-tetrahydrobenzo[f] oxazolo[3,2d] $[1,4]$ diazepin-6(5H)-one) is an anxiolytic oxazolo-BZD that is structurally similar to oxazolam and cloxazolam [17].

\section{Pharmacokinetic Considerations}

After the oral administration of mexazolam, its active metabolites chloronordiazepam (CND) 
and chloroxazepam (COX), but not the unaltered drug, are detected in the blood. Mexazolam appears to be hydroxylated and conjugated in the liver through two different metabolic pathways (BZD-type [active metabolites] and benzophenone-type [inactive metabolites]) [16]. The time-to-peak plasma concentration for $\mathrm{CND}$ is $1-2 \mathrm{~h}$ following the administration of a single oral dose of mexazolam. A bi-compartmental pharmacokinetic model can be applied, with a biphasic plasma concentration curve, and first and second half-lives of 1.4 and $76 \mathrm{~h}$, respectively. Both $\mathrm{CND}$ and COX are mainly bound to plasma proteins ( $>90 \%)$, and the majority of the drug is excreted via bile $(<10 \%$ of an oral dose of mexazolam is eliminated as metabolites in urine) [16, 18]. The active metabolite, COX, represents more than $50 \%$ of the total amount of excreted metabolites, and is essentially found as a conjugate [16, 19]. Although only small amounts of mexazolam may be found in breast milk, the drug should not be given to breast feeding mothers $[16,19]$.

\section{Preclinical Studies}

Like cloxazolam and oxazolam, mexazolam demonstrated a broad safety profile and very low acute toxicity in preclinical studies. The results of studies conducted in rats reported that the subacute or chronic administration of mexazolam showed no toxic reactions below a dosage of $125 \mathrm{mg} / \mathrm{kg}[16,20]$. Teratogenicity and mutagenic tests were negative, and preclinical animal studies did not indicate any harmful effects for mexazolam during pregnancy $[16,17]$. However, the safety of this drug in humans has not been established during pregnancy.

Preclinical studies showed that mexazolam was 2-3 times more effective than diazepam and cloxazolam at improving performance in conflict behavior or lever-pressing tests (regarded as a measure of an antianxiety effect) $[21,22]$. Mexazolam was also shown to inhibit megimide-induced convulsions in mice [23]. In electrophysiological tests using cats, mexazolam showed a stronger action than those of cloxazolam and diazepam with respect to the cerebral marginal gyrus, especially the amygdaloid nucleus, which plays important roles in instinctive behavior and emotional activity. Concomitant side effects such as drowsiness, muscle relaxation, and motor ataxia are weaker than those of diazepam and cloxazolam [23]. The results of preclinical studies also showed that mexazolam appears to have a more potent antianxiety effect than cloxazolam and diazepam, while causing less muscular relaxation, sedation, and ataxia $[23,24]$.

\section{Clinical Studies}

Mexazolam has been extensively studied for the treatment of anxiety disorders, and the results of a number of clinical trials have been published. Phase I and II trials reported that mexazolam was associated with a reduction in anxiety symptoms and few side effects [25-30]. A phase III, multicenter, double-blind, randomized study of 165 patients with psychosomatic disorders or neurosis confirmed the antianxiety effect of mexazolam (final global improvement rates of $75.6 \%$ and $63.8 \%$ with mexazolam and oxazolam, respectively) [31]. Based on these results, the authors concluded that mexazolam appeared to be a more effective drug than oxazolam.

A number of additional studies have since been conducted to determine the clinical efficacy and tolerability of mexazolam. 


\section{Clinical Efficacy}

Katsunuma et al. [23] conducted a phase II open-label uncontrolled study in an adult/ elderly population of 29 patients (age range 44-91 years) diagnosed with neurosis, anxiety, insomnia, indefinite complaints, and psychosomatic diseases from the Geriatric Department of Tokyo Medical College Hospital. It should be noted that the dosing schedule for mexazolam was different in this study compared with the currently approved dosing schedule [16]. Initially, mexazolam was administered at a dose of $1 \mathrm{mg}$ TID, followed by uptitration to $2 \mathrm{mg}$ TID according to conditions. According to the evaluation of treatment effectiveness (excellent, good, fair, and poor), 15 of 29 patients (51.7\%) classified their treatment as excellent, 9 patients (31.0\%) considered their treatment to be good, and 5 patients $(17.2 \%)$ considered it to be fair. The overall efficacy rate (proportion of patients with a treatment effectiveness of excellent or good) was $82.7 \%$, and efficacy rates between younger (aged $\leq 59$ years) and elderly ( $\geq 60$ years) patients were similar (85.7\% vs. 81.8\%) [23].

Ohara et al. [32] conducted a prospective, multicenter open-label, uncontrolled study in 44 Japanese patients ( 21 males and 23 females) with diseases that fell under the category of "neurosis". Patients were initially treated with mexazolam at a daily dosage of $1 \mathrm{mg}$, which could be adjusted, as required, based on the condition of the disease. The Hamilton Anxiety Rating Scale (HAM-A), and final global improvement rates (assessment of efficacy) were evaluated at baseline and weekly intervals until the end of the treatment period (4 weeks). Final global improvement rates were "markedly improved" in 15 patients (34.1\%), "improved" in 14 patients (31.8\%), "slightly improved" in 10 patients (22.7\%), and were rated as "no change" in 4 patients (9.1\%). Mexazolam rapidly improved the mean HAMA score by reducing it from 22.0 points at baseline to 14.5 points at Week $1,10.0$ points at Week 2, 9.0 points at Week 3, and 7.0 points at Week 4. One patient dropped out of the study due to a diagnosis of hysteria [32].

Vaz-Serra [33] conducted a 4-week doubleblind study that compared the efficacy and safety of mexazolam [1 mg twice daily (BID)] and bromazepam (3 mg BID) in 60 patients. Efficacy was assessed at 0, 14, and 28 days using the HAM-A scale and investigator-reported global clinical evaluation of the patient. Significant reductions in HAM-A scores were seen in both treatment groups at Days 14 and 28 (Fig. 1). Furthermore, the reductions were greater in patients treated with mexazolam. These results appeared to translate to an overall improvement in patient functioning for both study drugs, although this improvement was statistically higher with mexazolam [33].

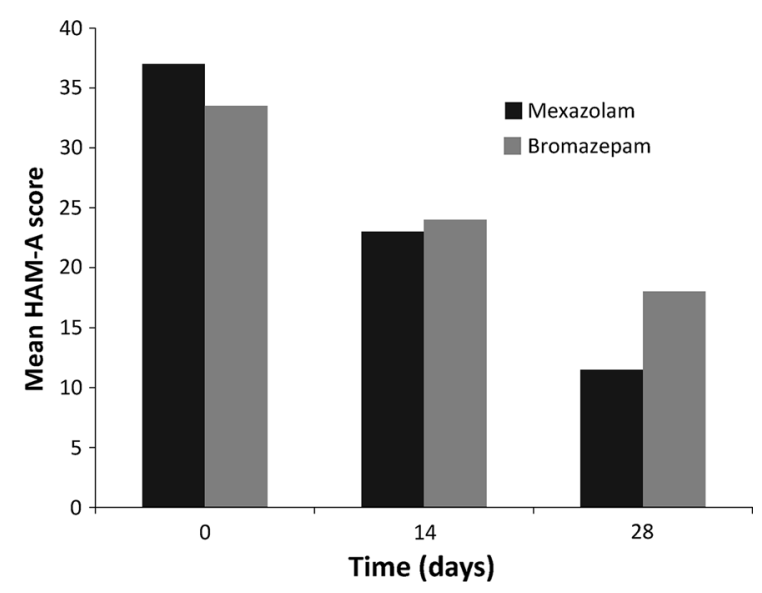

Fig. 1 Mean Hamilton Anxiety Rating Scale (HAM-A) scores at Days 0, 14, and 28 during treatment with mexazolam or bromazepam. Lambda de Wilks $=0.478$, $F$ test $=25.107 ; P=0.000$. Adapted with permission from Vaz-Serra A. Estudo clínico com dupla ocultação comparando mexazolam com bromazepam. Psiquiatría clínica. 1993;14(2):77-84 
Table 1 Percentage reductions in the frequency and severity of somatic anxiety symptoms after 28 days of treatment with mexazolam in patients with anxiety disorders. Reproduced with permission from Vieira Coelho and Garrett [17]

\begin{tabular}{lll}
\hline Symptoms & $\begin{array}{l}\text { Percent reduction } \\
\text { in frequency } \\
\text { (always or } \\
\text { frequent) }\end{array}$ & $\begin{array}{l}\text { Percent reduction } \\
\text { in severity } \\
\text { (incapacitating or } \\
\text { severe) }\end{array}$ \\
\hline Asthenia & 83.3 & 78.6 \\
$\begin{array}{c}\text { Daytime } \\
\text { sedation }\end{array}$ & 53.1 & 33.3 \\
Headache & 92.2 & 86.1 \\
Distractedness & 79.3 & 81.2 \\
Irritability & 88.3 & 95.9 \\
Restlessness & 89.6 & 91.5 \\
Dry mouth & 81.0 & 85.3 \\
Increased & 63.6 & 66.6 \\
appetite & & 87.5 \\
Decreased & 83.3 & \\
appetite & & 95.7 \\
$\begin{array}{c}\text { Palpitations } \\
\text { Chest }\end{array}$ & 89.9 & 95.7 \\
tightness & 94.6 & \\
\hline
\end{tabular}

In addition to the assessment of HAM-A, the investigator-reported global clinical evaluation of the patients at Day 14 showed that 16 of the 25 patients $(64.0 \%)$ treated with mexazolam had a significant improvement, compared with 8 of the 24 patients (33.3\%) treated with bromazepam [33]. At study end (Day 28), the majority of patients treated with mexazolam (92.0\%) showed a significant improvement, compared with $58.3 \%$ of patients treated with bromazepam.

Vieira Coelho and Garrett [17] published a prospective, multicenter, open-label, uncontrolled phase IV study that was conducted in the psychiatry departments of nine Portuguese hospitals. A total of 409 patients (aged 18-60 years) with a diagnosis of anxiety disorder (according to DSM-III-R classification) were enrolled; the majority of these patients had generalized anxiety disorder. Patients received mexazolam 2 or $3 \mathrm{mg}$ /day (according to their baseline HAM-A score) for 28 days, and clinical observations were recorded on Days 0, 7, and 28. Efficacy was evaluated by the HAM-A scale and a list of 27 symptoms was used to assess the frequency and severity of somatic anxiety components. Mexazolam significantly reduced mean $( \pm$ SD) HAM-A scores from $28.1 \pm 0.38$ at baseline, to $19.6 \pm 0.39$ at Day 7, and $12.1 \pm 0.4$ at Day 28 (both $P<0.05$ vs. baseline) [17]. Mexazolam also reduced the 27 somatic anxiety symptoms that were evaluated. Greater percent reductions in frequency and severity were noted for asthenia, headache, distractedness, irritability, restlessness, palpitations, and chest tightness (Table 1). At study end, the improvement in symptoms was considered to be "excellent" or "very good" in $65 \%$ of patients, "fair" in $24 \%$, and "null" in 11\% [17].

Vaz-Serra et al. [34] compared the anxiolytic effects of mexazolam and alprazolam in patients with generalized anxiety disorder (DSM-IV criteria). This 5-week multicenter, double-blind, randomized, parallel-group trial randomly assigned 64 patients to fixed doses of mexazolam $1 \mathrm{mg}$ TID or alprazolam $0.5 \mathrm{mg}$ TID for 1 week, followed by a 2-week period where the dosages of both drugs could be reduced according to therapeutic response. On Day 21, a 1-week taper period was initiated, which was followed by a 1-week treatment-free follow-up period. The two main measures of efficacy were the assessment of the HAM-A and Clinical Global Impression (CGI) scales, which were evaluated at baseline (Day 0), Day 7, Day 21, Day 28, and Day 35. A significant anxiolytic 


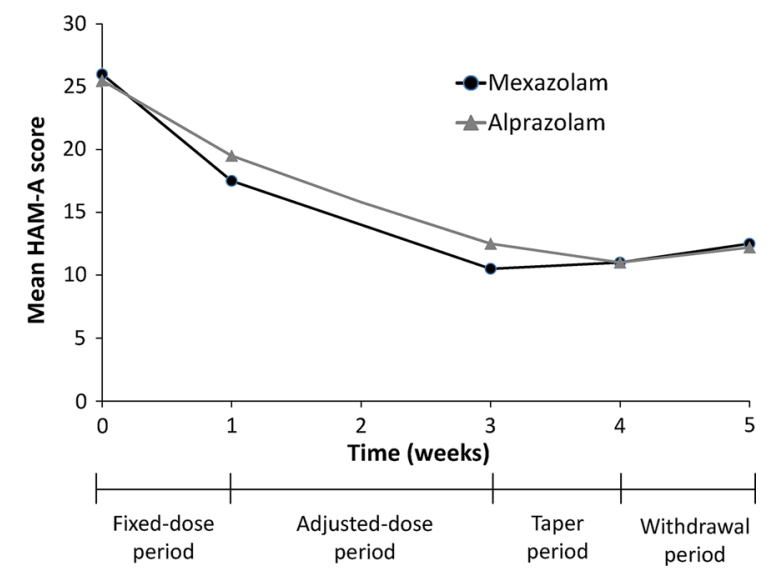

Fig. 2 Changes from baseline in the Hamilton Anxiety Rating Scale (HAM-A) mean score during treatment with mexazolam or alprazolam in patients with generalized anxiety disorder (within-group analysis: $P<0.0001$, Friedman non-parametric test; between-group comparison: $P>0.05$, analysis of variance). Adapted with permission from Vaz-Serra A et al. [33]

effect was seen in both treatment groups at study end, as seen by a significant reduction from baseline in the mean HAM-A score of 16.28 with mexazolam $(P<0.0001$ vs. baseline) and 14.2 with alprazolam $(P<0.0001$ vs. baseline; Fig. 2) [34]. On Day 7, the proportion of HAM-A responders (a decrease from baseline in HAM-A global scores of $\geq 50 \%$ ) was $35.5 \%$ with mexazolam and $23.3 \%$ with alprazolam; HAM-A responder rates were further increased to $80.0 \%$ with mexazolam and $70.0 \%$ with alprazolam on Day 21 [34]. Although these results appeared to be higher in the mexazolam group, the between-group differences between mexazolam and alprazolam were not statistically significant. Mexazolam and alprazolam were also associated with significant mean reductions from baseline in the mean CGI-disease severity score (CGI-DSS; 2.66 and 2.44, respectively; both $P<0.0001$ vs. baseline), with no significant between-group differences [34]. Furthermore, the beneficial anxiolytic effects of mexazolam and alprazolam were sustained during the tapering and withdrawal periods, as shown by the CGIDSS mean scores remaining significantly lower than baseline in both treatment groups. As with the HAM-A score, CGI-global improvement score (CGI-GIS) responder rates (the proportion of patients who were "highly improved" or "moderately improved") were higher in the mexazolam group compared with the alprazolam group at the end of weeks 1 (54.8\% vs. $46.7 \%)$ and 3 (96.7\% vs. $86.7 \%)$; however, these differences did not reach statistical significance [34].

\section{Clinical Tolerability}

With regard to tolerability, Katsunuma et al. [23] reported a mild degree of drowsiness in 5 of the 29 patients $(17.2 \%)$. In addition, no falls were reported.

In the study conducted by Ohara et al. [32], mexazolam-related side effects were reported in 11 of the 44 patients (25.0\%); drowsiness was the most frequently reported side effect (7 patients). No serious side effects requiring a reduction in dosage were reported [32]. In addition, no abnormalities in clinical laboratory tests (including hematological and urinalysis findings) were seen in the 24 patients who underwent testing before and after the completion of mexazolam therapy.

In the study published by Vaz-Serra [33] in 1993, no side effects were reported in the majority of patients; side effects were generally minor in terms of severity, and did not alter the general state of the patient. In a clinical assessment of general symptoms that could be considered to be side effects, no significant changes in these symptoms were seen prior to versus during mexazolam therapy. Asthenia and sedation were the symptoms most frequently reported during the study. Vaz-Serra [33] also assessed memory changes, which are often 
adversely affected by the use of BZDs. Memory changes were evaluated using the digit span test (the ability of a patient to fix and immediately reproduce a sequence of five numbers, repeating this procedure three times in each observation) and a questionnaire to evaluate memory retention and recall [33]. The results showed no detrimental alterations in patient memory; in fact, a significant improvement in the digit span test was seen for mexazolam versus bromazepam $(P=0.015)$. This finding suggests that the improvement in anxiety levels may lead to improvements in the capacity to retain and subsequently recall messages.

In the phase IV study conducted by Vieira Coelho and Garrett [17], the overall incidence of side effects was $11.7 \%$ at Day 28 , with the most frequent event being daytime sedation $(7.5 \%$ of patients). The investigator-rated intensity of side effects was considered to be inconsequential in $65 \%$ of patients and mild in $31 \%$; side effects that interfered with normal daily activity were reported in $4 \%$ of patients. The effect of mexazolam on cognition and memory performance was evaluated by an objective test [Negative Symptom Rating Scale: B-cognition III x-Memory (NSRS)], which showed no impairment of cognitive function during the study. Similar to the Vaz-Serra [33] 1993 study, enhanced performance on the memory test, demonstrated by significant improvements from Day 0 in NSRS scores at Days 7 and 28 (both $P<0.05$ ) was observed in patients receiving mexazolam and authors again indicated that memory improvement was likely to be related to the anxiolytic effect of mexazolam (Fig. 3) [17].

In 2001, the study by Vaz-Serra et al. [34] reported no clinically important differences in tolerability between mexazolam and alprazolam. Adverse events in both treatment groups were mild to moderate in intensity and

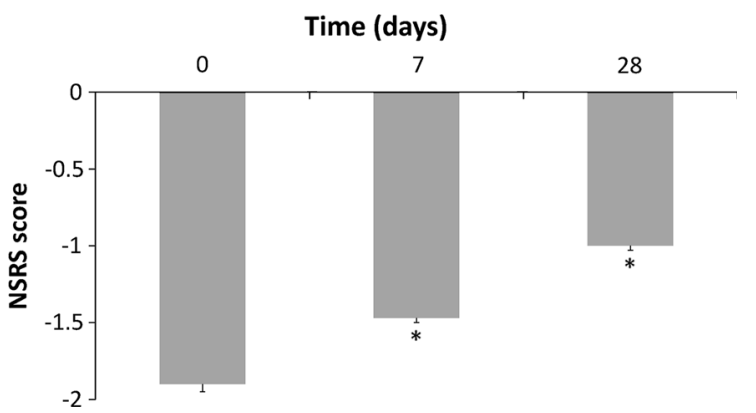

Fig. 3 Mean scores on the Negative Symptom Rating Scales: B-Cognition III (x-Memory) (NSRS). Word recall was assessed as follows: a all five words recalled without difficulty (rating of 0); b four words or possible recall of fifth word after prompting $(-1$ to -2$)$; $\mathbf{c}$ two or three words recalled, prompting notwithstanding ( -3 to -4$)$; $\mathbf{d}$ no words recalled or possible recall of one word after prompting $(-5$ to -6$) .{ }^{*} P<0.05$ (Wilcoxon test); significance between consecutive evaluations. Adapted with permission from Vieira Coelho and Garrett [17]

consistent with the well-known tolerability profile of BZDs. The most commonly reported event was drowsiness, which occurred in 9.7\% and $10.0 \%$ of patients receiving mexazolam and alprazolam, respectively.

In addition to the safety issues described above, BZD use has been associated with impaired psychomotor function, resulting in the disruption of tasks that require sustained attention, vigilance, speed, and accuracy [3537]. Preclinical studies indicate that although the anxiolytic properties of mexazolam are similar to those of diazepam and cloxazepam, mexazolam produces less sedation, ataxia, and muscular relaxation [38]. This suggests that mexazolam may be associated with fewer deleterious effects on psychomotor performance and cognitive function than other BZDs. It was in this context that Silveira et al. [18] conducted a double-blind, randomized, two-way crossover, placebocontrolled study in 33 healthy adult volunteers to assess the effect of a single 1-mg oral dose of mexazolam on psychomotor performance. Psychomotor performance was 
Table 2 Leeds Psychomotor Test Battery (critical flicker fusion threshold; motor reaction time; recognition reaction time; total reaction time) and car-driving simulation (total time score; best lap time) results before and after a single oral dose of mexazolam $1 \mathrm{mg}$ or placebo in healthy volunteers

\begin{tabular}{|c|c|c|c|c|}
\hline & \multicolumn{2}{|l|}{ Placebo } & \multicolumn{2}{|l|}{ Mexazolam } \\
\hline & Pre-dose & Post-dose & Pre-dose & Post-dose \\
\hline \multicolumn{5}{|c|}{ Leeds Psychomotor Test Battery results } \\
\hline CFF threshold $(\mathrm{Hz})$ & $29.03 \pm 2.94$ & $29.06 \pm 3.01$ & $29.62 \pm 3.01$ & $28.85 \pm 3.35$ \\
\hline RRT (ms) & $379.74 \pm 44.29$ & $385.87 \pm 39.59$ & $381.18 \pm 56.64$ & $378.42 \pm 36.66$ \\
\hline MRT (ms) & $209.25 \pm 39.92$ & $208.77 \pm 43.90$ & $210.35 \pm 37.24$ & $205.05 \pm 33.58$ \\
\hline TRT (ms) & $588.99 \pm 67.00$ & $594.64 \pm 63.22$ & $591.52 \pm 74.58$ & $583.47 \pm 51.37$ \\
\hline \multicolumn{5}{|c|}{ Car-driving simulation results } \\
\hline TTS (s) & $745.69 \pm 208.70$ & $708.61 \pm 125.45^{*}$ & $724.70 \pm 90.73$ & $707.19 \pm 75.15^{*}$ \\
\hline $\operatorname{BLT}(s)$ & $44.73 \pm 9.81$ & $43.02 \pm 6.07^{*}$ & $43.69 \pm 4.12$ & $43.02 \pm 3.19^{*}$ \\
\hline
\end{tabular}

Data shown are mean \pm SD. Data from Silveira et al. [18]

$B L T$ best lap time, $C F F$ critical flicker fusion, $H z$ Hertz, $M R T$ motor reaction time, ms millisecond, $R R T$ recognition reaction time; $s$ second, $T R T$ total reaction time

${ }^{*} P<0.05$ versus pre-dose values

evaluated using the Leeds Psychomotor Test (LPT) Battery [comprising the critical flicker fusion (CFF) threshold evaluating visual information processing time and the choice reaction time with its three componentsrecognition reaction time (RRT), motor reaction time (MRT), and total reaction time (TRT)] and a car-driving simulation (CDS). After the administration of mexazolam, changes (prevs. $3 \mathrm{~h}$ post-administration) in the LPT Battery and CDS results were not significantly different compared with placebo (Table 2) [18]. CDS results indicated a significant improvement for both placebo and mexazolam, which indicated a learning effect induced by repetition of the tests (Table 2). Regarding adverse events, three subjects reported three events, one (somnolence) in the mexazolam group.

Ferreira et al. [24] conducted a multicenter, randomized, double-blind, parallel-group, placebo-controlled clinical trial in 60 outpatients with generalized anxiety disorder
(DSM-IV criteria) who received treatment with mexazolam $1 \mathrm{mg}$ TID $(n=32)$ or placebo $(n=28)$ for 21 days (Visit 1 , screening and start of placebo run-in; Visit 2, Day 0; Visit 3, Day 7; Visit 4, Day 21). The effects of mexazolam on psychomotor performance (as assessed by the LPT Battery; primary endpoint) and clinical status (HAM-A and CGI) were evaluated.

No statistically significant differences were seen between mexazolam and placebo with regard to the LPT Battery results (CFF, RRT, MRT, and TRT) [24]. Regarding HAM-A assessment, significant improvements in total and somatic scores were seen with mexazolam versus placebo at Day 7, but not at Day 21 . However, it should be noted that sample size was estimated on the basis of the CFF parameter, and that high-dose regimens of well-established drugs such as diazepam have also shown this phenomenon. The most frequently reported adverse event was 
drowsiness, which occurred in eight patients receiving mexazolam and one patient receiving placebo.

\section{DISCUSSION}

To our knowledge this is the first review of articles to be conducted for mexazolam. Despite the wide availability of BZDs and their rapid onset of action, selective serotonin reuptake inhibitors (SSRIs) and serotoninnorepinephrine reuptake inhibitors (SNRIs) are the recommended first-line agents for anxiety due to their favorable risk-benefit ratio, with BZDs used as an adjunct to SSRIs and SNRIs in the first few weeks of treatment (for control of short-term anxiety or when deemed necessary by the clinician [6]. BZDs can be a useful adjunct, as the anxiolytic effect of SSRIs and SNRIs has a delayed onset of action of 2-4 weeks, and, in some cases, up to 6-8 weeks); however, we have not found in the literature any papers describing or evaluating this specific use of mexazolam in the short-term treatment of anxiety.

Globally, BZDs have a good safety record and should be used with a regular dosing regimen but are not effective in acute stress disorder, in patients with comorbid depression, or in those with obsessive-compulsive and related disorders [6].

Mexazolam is not mentioned specifically in the latest European guidelines, probably because it is not available in many European countries (nevertheless, it is mentioned in national guidelines of countries where the drug is available, for example, Portugal) [39]. Mexazolam is approved in some other countries in Europe, Central America, Africa, and Asia. In this sense, it has become important to conduct a review of the clinical characteristics of mexazolam.
In our research for this manuscript, we found a lack of recent clinical data on mexazolam, and that is the main limitation of this review. This lack of recent clinical data is probably due to the fact that mexazolam has had an established therapeutic value in the management of anxiety for many years, with no expected potential use in other indications, and thus no further clinical trials have been conducted. Nevertheless, the clinical use of mexazolam and BZDs in general is valuable; mexazolam has been shown to be useful in the management of anxiety with or without psychoneurotic conditions when the disorder is severe, disabling, or subjecting the individual to extreme distress [17].

The efficacy and tolerability of mexazolam in patients (including the elderly) with disorders associated with anxiety appears to have been previously well established based on the clinical data that is available in the literature $[24,26$, 31-33]. Following on from this initial evidence, a number of additional multicenter and comparative phase III/IV clinical trials have been subsequently conducted to confirm the efficacy and safety of mexazolam for this indication [17, 18, 24, 31-34, 40-46].

The multicenter, phase IV trial by Vieira Coelho and Garrett [17] confirmed the efficacy of mexazolam in the treatment of anxiety, demonstrating a rapid anxiolytic effect that resulted in a 30\% reduction in HAM-A scores after 1 week of treatment; further reductions were seen at Day 28. Somatic symptoms commonly associated with anxiety were also reduced by approximately 80-94\% following treatment with mexazolam [17]. Based on these findings, the authors concluded that mexazolam was an effective anxiolytic with a good safety profile (no impairment of cognitive function or major sedative side effects was reported). 
Two comparative studies $[33,34]$ were conducted after the publication of an initial comparative study of mexazolam versus oxazolam by Suzuki et al. [31] in 1980. In 1993, Vaz-Serra [33] concluded that mexazolam provided a superior anxiolytic effect versus bromazepam. With regard to tolerability, no significant treatment-related side effects were seen with either study drug, particularly detrimental changes in memory/cognition (a finding that was later reinforced by Vieira Coelho and Garrett [17]).

A second comparative study was conducted by Vaz-Serra et al. [34] in 2001, which concluded that both mexazolam and alprazolam were well tolerated. Daytime sedation was the most frequently reported side effect ( $9.7 \%$ of patients) associated with the use of mexazolam. The frequency of daytime sedation in the mexazolam group for this study was similar to that observed in Vieira Coelho and Garrett [17] (7.5\%), and lower than that observed in the studies by Katsunuma et al. [23] and Ohara et al. [32] (although the mexazolam dosages used in these two studies were higher [ 6 and $4 \mathrm{mg} /$ day, respectively] and they were not double-blind, placebo-controlled studies). With regard to efficacy, Vaz-Serra et al. [34] concluded that mexazolam appeared to be associated with higher HAM-A and CGI-GIS responder rates versus alprazolam, although the between-group differences did not reach statistical significance. The study also showed that mexazolam had a fast onset of action (significant reductions in anxiety scores were seen in the first week of treatment) and a sustained effect throughout the study, including the tapering and treatment-free follow-up periods [34]. This short latency of the anxiolytic effect for mexazolam is clinically relevant, because it enables the rapid and consistent control of symptoms.
The impairment of psychomotor performance and cognitive performance are two major complaints associated with BZD therapy, particularly during long-term use [4043]. In the study by Silveira et al. [18], LPT Battery and CDS results revealed no statistically significant differences between mexazolam and placebo. These findings are in accordance with the good tolerability profile of mexazolam in previously reported clinical trials, particularly the low incidence of drowsiness and sedation [34]. The results of this study are of clinical relevance because there was an absence of adverse psychomotor effects at the time when such effects are most likely to occur during treatment with mexazolam or other BZDs. In contrast to mexazolam, significant reductions in speed of visual information processing (measured by CFF threshold) have been seen with other BZDs (diazepam, chlordiazepoxide, oxazepam, lorazepam, and nitrazepam) [44, 45], which indicates a negative effect on overall central nervous system activity. The use of these BZDs as antianxiety treatments has also been associated with side effects such as forgetfulness, impairment of psychomotor function, fatigue, and reductions in attention, cognitive ability, and overall psychological skills. Based on the results reported by Silveira et al. [18] and the fact that mexazolam $1 \mathrm{mg}$ is equivalent to diazepam $1 \mathrm{mg}$ [46], it is possible that mexazolam therapy may be associated with few adverse effects on cognitive function and little or no impairment of daily activities. Furthermore, Silveira et al. [18] showed that mexazolam did not affect learning ability (no significant differences compared with placebo).

Finally, the study by Ferreira et al. [24] showed that there was an absence of disruptive effects on psychomotor performance following the administration of clinically effective anxiolytic dosages of 
mexazolam. Mexazolam demonstrated a good tolerability profile in this study, which was in agreement with the absence of psychomotor and cognitive performance impairment; this in turn may lead to better compliance. However, the small sample size of the study does not allow accurate conclusions to be drawn.

Although the clinical evidence is limited, it would appear that mexazolam is at least as effective (e.g., alprazolam) or even more effective (e.g., bromazepam and oxazolam) than other BZDs and better tolerated and so it may have the potential for an increased role in the treatment of anxiety and other specific indications.

Other considerations for use of mexazolam over other BZDs and other treatment used in the management of anxiety include its relative cost effectiveness. Currently, there are no pharmacoeconomic data for mexazolam. Until such data are available, one can substantiate its clinical use by other important parameters such as clinical efficacy and tolerability.

\section{CONCLUSION}

To our knowledge, this is the first published review article that provides an overview on the use of mexazolam in the treatment of anxiety. Mexazolam is indicated for the management of anxiety with or without psychoneurotic conditions. In addition to its clinical efficacy, mexazolam is also associated with a rapid onset of its anxiolytic effect. The limited clinical evidence that is available to date suggests that mexazolam is more effective than bromazepam and oxazolam, and is at least as effective as alprazolam. Furthermore, mexazolam does not appear to be associated with adverse effects on the psychomotor and cognitive performance of anxious patients, which may lead to better compliance and additional specific indications for this drug. Additional, larger, well-controlled studies are required to further establish and directly compare mexazolam's efficacy and tolerability with other BZDs for the management of anxiety.

\section{ACKNOWLEDGMENTS}

Sponsorship for the article processing charges was received from Bial - Portela \& $C^{a}$, S.A., Portugal. Editorial assistance in the preparation of this manuscript was provided by Maxwell Chang and Mary Hines of inScience Communications, Springer Healthcare. This assistance was funded by Bial - Portela \& $C^{a}$, S.A., Portugal. The authors would also like to thank Nuno Palma PhD, Manager of Bioinformatics and Statistics at Bial Portela \& $C^{a}$, S.A., for providing the chemical structure of mexazolam.

Both named authors meet the ICMJE criteria for authorship for this manuscript, take responsibility for the integrity of the work as a whole, and have given final approval to the version to be published.

Conflict of interest. Dr. Fernandes is a medical manager at Bial - Portela \& $C^{a}$, S.A. Dr. Moreira has been a consultant for and has received honoraria as a speaker for Bial - Portela $\& C^{a}$, S.A.

Compliance with ethics guidelines. The analysis in this article is based on previously conducted studies, and does not involve any new studies of human or animal subjects performed by any of the authors.

Open Access. This article is distributed under the terms of the Creative Commons Attribution Noncommercial License which permits any noncommercial use, distribution, 
and reproduction in any medium, provided the original author(s) and the source are credited.

\section{REFERENCES}

1. Kessler RC, Berglund P, Demler O, Jin R, Merikangas $\mathrm{KR}$, Walters EE. Lifetime prevalence and age-ofonset distributions of DSM-IV disorders in the National Comorbidity Survey Replication. Arch Gen Psychiatry. 2005;62(6):593-602.

2. Kessler RC, Chiu WT, Demler O, Merikangas KR, Walters EE. Prevalence, severity, and comorbidity of 12-month DSM-IV disorders in the National Comorbidity Survey Replication. Arch Gen Psychiatry. 2005;62(6):617-27.

3. Kessler RC, Wang PS. The descriptive epidemiology of commonly occurring mental disorders in the United States. Annu Rev Public Health. 2008;29: 115-29.

4. Kessler RC, Angermeyer M, Anthony JC, et al. Lifetime prevalence and age-of-onset distributions of mental disorders in the World Health Organization's World Mental Health Survey Initiative. World Psychiatry. 2007;6(3):168-76.

5. American Psychiatric Association. Anxiety Disorders, Section II: Diagnostic Criteria and Codes. Diagnostic and Statistical Manual of Mental Disorders, Fifth Edition, DSM-5, 5th edn. Arlington: American Psychiatric Association; 2013. doi:10.1176/appi.books.9780890425596.257491.

6. Bandelow B, Sher L, Bunevicius R, et al. Guidelines for the pharmacological treatment of anxiety disorders, obsessive-compulsive disorder and posttraumatic stress disorder in primary care. Int J Psychiatry Clin Pract. 2012;16(2):77-84.

7. National Institute for Health and Clinical Excellence. Generalised anxiety disorder and panic disorder (with or without agoraphobia) in adults National Institute for Health and Clinical Excellence. 2011. http://publications.nice.org.uk/ generalised-anxiety-disorder-and-panic-disorderwith-or-without-agoraphobia-in-adults-cg113. Accessed 29 July 2013.

8. National Institute of Mental Health. Anxiety disorders. U.S. Department of Health and Human Service, National Institutes of Health. 2009. http:// www.nimh.nih.gov/health/publications/anxietydisorders/index.shtml. Accessed 29 July 2013.

9. Mathers DA, Yoshida $H$. The benzodiazepine triazolam: direct and GABA depressant effects on cultured mouse spinal cord neurons. Eur J Pharmacol. 1987;139(1):53-60.

10. Obata T, Yamamura HI. The effect of benzodiazepines and beta-carbolines on GABAstimulated chloride influx by membrane vesicles from the rat cerebral cortex. Biochem Biophys Res Commun. 1986;141(1):1-6.

11. Lader M. Benzodiazepines revisited-will we ever learn? Addiction. 2011;106(12):2086-109.

12. Breimer DD, Jochemsen $\mathrm{R}$, von Albert $\mathrm{HH}$. Pharmacokinetics of benzodiazepines. Short-acting versus long-acting. Arzneimittelforschung. 1980; 30(5a):875-81.

13. Riss J, Cloyd J, Gates J, Collins S. Benzodiazepines in epilepsy: pharmacology and pharmacokinetics. Acta Neurol Scand. 2008;118(2):69-86.

14. Rynn MA, Brawman-Mintzer O. Generalized anxiety disorder: acute and chronic treatment. CNS Spectr. 2004;9(10):716-23.

15. Longo LP, Johnson B. Addiction: Part I. Benzodiazepines-side effects, abuse risk and alternatives. Am Fam Physician. 2000;61(7):2121-8.

16. BIAL - Portela \& $C^{\mathrm{a}}$ S.A. Sedoxil, $1 \mathrm{mg}$, tablet, Summary of Product Characteristics. S. Mamede do Coronado, Portugal; 2009.

17. Vieira Coelho MA, Garrett J. Mexazolam in anxiety disorders: results of a multicenter trial. Adv Ther. $1997 ; 14(3): 125-33$.

18. Silveira P, Vaz-da-Silva M, Dolgner A, Almeida L. Psychomotor effects of mexazolam vs placebo in healthy volunteers. Clin Drug Investig. 2002; 22(10):677-84.

19. Hasegawa M, Esumi Y, Takaichi M, Kakurai Y, Nampo T. Studies on absorption, distribution, and excretion of CS-386. Tokai Laboratories, Daiichi pure chemicals Co., Ltd. (data on file).

20. Masuda H, Kimura K, Yamashita K, Kanamaru M. Toxicity studies of CS-386. Acute, subacute and chronic toxicity studies in experimental animals. Ann Rep Snkyo Res Lab. 1978;30:175-92.

21. Kamioka T, Nakayama I, Akiyama S, Takagi H. Effects of oxazolam, cloxazolam, and CS-386, new anti-anxiety drugs, on socially induced suppression and aggression in pairs of monkeys. Psychopharmacology. 1977;52(1):17-23.

22. Takagi H. Pharmacology of a benzodiazepinoaxazole derivative, a new minor tranguilizer. Ann Rep Snkyo Res Lab. 1971;23: 1-53. 
23. Katsunuma H, Shimoto Y, Senba K, Fujita R, Sakurai Y. Clinical trials of the anti-anxiety drug CS-386 (mexazolam) in the field of geriatrics. Geriatr Med. 1981;19(1):128-34.

24. Ferreira L, Figueira ML, Bessa-Peixoto A, et al. Psychomotor and anxiolytic effects of mexazolam in patients with generalised anxiety disorder. Clin Drug Investig. 2003;23(4):235-43.

25. Fukumizu T. Clinical experience with the new minor tranquilliser CS-386. Basic Pharmacol Ther. 1978;6:156-63.

26. Hasegawa N. Clinical trials of CS-386 against psychosomatic diseases and neuroses. Basic Pharmacol Ther. 1978;6(4):165-70.

27. Oumi ST, Nakano S, Yamada S, Natsume M. The experience on clinical trials of the new minor tranquilizer CS-386. Drug Ther. 1978;11(11):20-6.

28. Sasaki DS, Kawakami M. Phase II clinical trials of the anti-anxiety drug CS-386 on patients with psychosomatic diseases and neuroses in the field of internal medicine. Drug Ther. 1978;11(11): 26-32.

29. Tsutsui S. Clinical trials of CS-386. Shinryo to Shinyaku. 1978;15(5):137-40.

30. Yasuro H, Reijiro W. Clinical pharmacology of CS386, tranquilliser (phase I study). Med Consult New Rem. 1979;16:984-1000.

31. Suzuki J, Namiki M, Kawakami K. Clinical evaluation of a psychotropic drug, mexazolam in psychosomatics and neurosis: a double-blind trial with oxazolam. Jpn J Psychosom Med. 1980;20:301-16.

32. Ohara K, Suzuki Y, Kawaguchi K. Clinical evaluation of mexazolam (Melex) in neuroses. Jpn J Clin Exp Med. 1987;64:263-6.

33. Vaz-Serra A. Estudo clinico com dupla ocultacao comparando mexazolam com bromazepam. Psiquiatr Clin. 1993;14(2):77-84.

34. Vaz-Serra A, Figueira ML, Bessa-Peixoto A, et al. Mexazolam and alprazolam in the treatment of generalized anxiety disorder. A double-blind, randomised clinical trial. Clin Drug Investig. 2001;21(4):257-63.
35. Hindmarch I, Haller J, Sherwood N, Kerr JS. Comparison of five anxiolytic benzodiazepines on measures of psychomotor performance and sleep. Neuropsychobiology. 1990;24(2):84-9.

36. Koelega HS. Benzodiazepines and vigilance performance: a review. Psychopharmacology. 1989;98(2):145-56.

37. Lader MH. Limitations on the use of benzodiazepines in anxiety and insomnia: are they justified? Eur Neuropsychopharmacol. 1999;9 (Suppl 6):S399-405.

38. Kobayashi H. Pharmacology of CS-386. Tokyo: Central Research Laboratories, Sankyo Co Ltd.

39. Departamento da Qualidade na Saúde. Norma da Direcção-geral da Saúde: Abordagem Terapêutica da Ansiedade e Insónia; 2011. N. 055/2011.

40. Barker MJ, Greenwood KM, Jackson M, Crowe SF. Cognitive effects of long-term benzodiazepine use: a meta-analysis. CNS Drugs. 2004;18(1):37-48.

41. Gorenstein C, Bernik MA, Pompeia S, Marcourakis T. Impairment of performance associated with long-term use of benzodiazepines. J Psychopharmacol. 1995;9(4):313-8.

42. Tonne $\mathrm{U}$, Hiltunen AJ, Vikander $\mathrm{B}$, et al. Neuropsychological changes during steady-state drug use, withdrawal and abstinence in primary benzodiazepine-dependent patients. Acta Psychiatr Scand. 1995;91(5):299-304.

43. Wittenborn JR, Flaherty CF Jr, McGough WE, Nash RJ. Psychomotor changes during initial day of benzodiazepine medication. Br J Clin Pharmacol. 1979;7(Suppl 1):69S-76S.

44. Hindmarch I. Critical flicker fusion frequency (CFF): the effects of psychotropic compounds. Pharmacopsychiatry. 1982;15(Suppl 1):44-8.

45. Hindmarch I. Antidepressant drugs and performance. Br J Clin Pract. 1982;19(Suppl):73-7.

46. Ito H, Kaizawa S, Miura S, et al. Comparison of mexazolam and diazepam in the treatment of neurosis: a double-blind controlled study. Clin Eval. 1981;9:319-54. 